\title{
Some new results for the Kumaraswamy modified Weibull distribution
}

\author{
Gauss M. Cordeiro ${ }^{1}$, Antonio C. R. Braga Junior ${ }^{2}$, Clarice G. B. Demétrio ${ }^{2}$, \\ Edwin M. M. Ortega ${ }^{2, a}$ and Rodrigo R. Pescim ${ }^{2}$ \\ ${ }^{1}$ Departamento de Estatística, Universidade Federal de Pernambuco-UFPE \\ Recife, Pernambuco, Brazil \\ ${ }^{2}$ ESALQ, Departamento de Ciências Exatas, Universidade de São Paulo-USP \\ Piracicaba, São Paulo, Brazil
}

\begin{abstract}
We study some mathematical properties of the Kumaraswamy modified Weibull distribution pioneered by Cordeiro et al. [4] not discussed by these authors. This model is quite flexible for analyzing positive data since it contains as special models some widely-known distributions, such as the Kumaraswamy Weibull, generalized modified Weibull, exponentiated Weibull, modified Weibull and Weibull distributions, among several others. The beauty and importance of this distribution lies in its ability to model both monotone and non-monotone failure rates that are quite common in lifetime problems and reliability. We derive a useful power series for the quantile function. Various new explicit expressions are derived for the asymptotes and shapes, skewness and kurtosis based on the quantile function, the ordinary, incomplete and factorial moments, generating function, and Bonferroni and Lorenz curves. We verify the performance of the maximum likelihood estimates of the model parameters by Monte Carlo simulation. The current model is modified to cope with possible longterm survivors in the data. An application is presented to show the potentiality of this model. A multivariate generalization is proposed.
\end{abstract}

Keywords: Cure rate model; Kumaraswamy distribution; Maximum likelihood estimation; Modified Weibull distribution; Multivariate generalization.

\section{Introduction}

The statistics literature is filled with hundreds of lifetime distributions. Recent developments focus on new techniques for building meaningful lifetime distributions. New extensions of the Weibull distribution were proposed to model bathtub-shaped failure rate since the Weibull distribution is not adequate for modeling phenomenon with non-monotone failure rates. Among these, we cite the exponentiated Weibull (EW) (Mudholkar et al., [11]), the additive Weibull (Xie and Lai, [15]), the extended Weibull (Xie et al., [16]) and the modified Weibull (MW) (Lai et al., [9]) distributions. The probability density function (pdf) of the three-parameter modified Weibull (MW) distribution (Lai et al., [9]), say $\operatorname{MW}(\alpha, \gamma, \lambda)$, is given by

$$
g(x)=\alpha x^{\gamma-1}(\gamma+\lambda x) \exp \left[\lambda x-\alpha x^{\gamma} \exp (\lambda x)\right], x>0,
$$

where $\alpha>0$ controls the scale of the distribution, $\gamma>0$ controls its shape and $\lambda \geq 0$ is a kind of accelerating factor in the imperfection time and it works as a factor of fragility in the survival of

\footnotetext{
aAddress for correspondence: Departamento de Cincias Exatas, USP, Av. Pdua Dias 11 - Caixa Postal 9, 13418-900 Piracicaba - São Paulo - Brazil. E-mail: edwin@usp.br
} 
the individual when the time increases. The cumulative distribution function (cdf) corresponding to (1.1) is given by

$$
G(x)=1-\exp \left[-\alpha x^{\gamma} \exp (\lambda x)\right]
$$

Providing a broader class of distributions is always precious for statisticians. Cordeiro et al. [4] proposed the five-parameter Kumaraswamy modified Weibull (KwMW) distribution which seems to be superior over the MW model for certain applications. A first positive point of this distribution is that it includes the MW model as a basic exemplar. A second one is that it includes as special cases some important lifetime models. The justification for the practicability of this model is based on its flexibility in accommodating bathtub-shape and unimodal forms of the hazard rate function (hrf). In this paper, we study some mathematical properties of the KwMW model not investigated by Cordeiro et al. [4] with the hope that it will attract wider applications in reliability, engineering and in other areas of research.

The cdf of the KwMW distribution (for $x>0$ ) is given by

$$
F(x)=1-\left\{1-\left[1-\exp \left\{-\alpha x^{\gamma} \exp (\lambda x)\right\}\right]^{a}\right\}^{b},
$$

where $\alpha>0, \gamma>0, \lambda \geq 0, a>0$ and $b>0$. The pdf corresponding to (1.3) reduces to

$$
\begin{aligned}
f(x)= & a b \alpha(\gamma+\lambda x) x^{\gamma-1} \exp \left[\lambda x-\alpha x^{\gamma} \exp (\lambda x)\right]\left[1-\exp \left\{-\alpha x^{\gamma} \exp (\lambda x)\right\}\right]^{a-1} \\
& \times\left\{1-\left[1-\exp \left\{-\alpha x^{\gamma} \exp (\lambda x)\right\}\right]^{a}\right\}^{b-1} .
\end{aligned}
$$

Hereafter, a random variable $X$ with pdf (1.4) is denoted by $X \sim \operatorname{KwMW}(a, b, \alpha, \gamma, \lambda)$.

The KwMW density function (1.4) allows for greater flexibility of its tails and can be widely applied in many areas of engineering and biology. We study the structural properties of this distribution because it extends some important distributions previously considered in the literature. In fact, for $\lambda=0$, it reduces to the Kumaraswamy Weibull (KwW) distribution. If $b=1$, it gives the generalized modified Weibull (GMW) distribution. If $\gamma=1$, in addition to $\lambda=0$, it yields the Kumaraswamy exponential (KwE)distribution. If $a=1$, in addition to $b=1$, we obtain the modified Weibull (MW) distribution. For $b=1$, in addition to $\lambda=0$, it gives the generalized Weibull (GW) distribution. This model is also known as the exponentiated Weibull (EW) distribution. If $\gamma=1$, in addition to $b=1$ and $\lambda=0$, it yields as special case the generalized exponential (GE) distribution. For $\gamma=2$, in addition $\lambda=0$ and $b=1$, it reduces to the generalized Rayleigh (GR) distribution. In Figure 1, we display some special models of the KwMW distribution, where the well-known sub-models not defined before are: the Kumaraswamy modified Rayleigh (KwMR), Kumaraswamy modified exponential (KwME), generalized modified Rayleigh (GMR), generalized modified Weibull (GMW), generalized modified exponential (GME), Kumaraswamy Rayleigh (KwR), modified Rayleigh (MR), modified exponential (ME), Rayleigh (R), Weibull (W) and exponential (E) distributions. This generalization is obtained by increasing the number of parameters compared to previously used distributions, this increase being the price to pay for adding more flexibility to the distribution. A further positive aspect of the KwMW model provides more flexibility for modeling skewed data.

The hrf of $X$ is given by

$$
\begin{aligned}
h(x)= & a b \alpha(\gamma+\lambda x) x^{\gamma-1} \exp \left[\lambda x-\alpha x^{\gamma} \exp (\lambda x)\right]\left[1-\exp \left\{-\alpha x^{\gamma} \exp (\lambda x)\right\}\right]^{a-1} \\
& \times\left\{1-\left[1-\exp \left\{-\alpha x^{\gamma} \exp (\lambda x)\right\}\right]^{a}\right\}^{-1}
\end{aligned}
$$




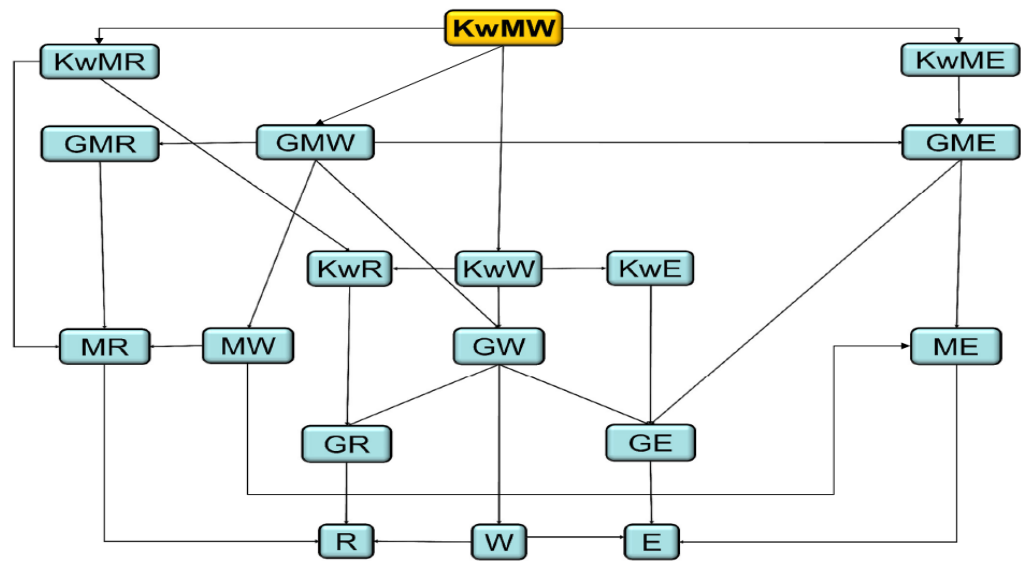

Fig. 1. Relationships of the KwMW special models.

respectively. Plots of the functions (1.4) and (1.5) for selected parameter values are given Cordeiro et al. [4].

Further, Cordeiro et al. [4] demonstrated that the KwMW density function can be expressed as a mixture of generalized modified Weibull (GMW) (Carrasco et al., [3]) densities. This is an important result to provide some structural properties of the KwMW model directly from those GMW properties. Unless otherwise stated, all the results derived in the paper are original. The rest of the paper is outlined as follows. In Section 2, we define the asymptotes and shapes. A useful expansion for the pdf of $X$ is reviewed in Section 3. A range of new KwMW structural properties is considered in Sections 4 to 7 . These include quantile moments, a power series for the quantile function, alternative explicit expressions for the ordinary, central, factorial and incomplete moments, cumulants, Bonferroni and Lorenz curves and generating function. For the first time, in Section 8, we propose the KwMW mixture model for survival data with cure fraction. In Section 9, we perform a simulation study for this broader class of distributions. An application to real data set in Section 10 shows the potentiality of the current model. A bivariate generalization of the KwMW model is presented in Section 11. Finally, some conclusions are addressed in Section 12.

\section{Asymptotes and shapes}

The asymptotes of equations (1.3), (1.4) and (1.5) as $x \rightarrow 0, \infty$ are given by

$$
\begin{aligned}
& F(x) \sim a\left[1-\exp \left\{-\alpha x^{\gamma} \exp (\lambda x)\right\}\right]^{a} \text { as } x \rightarrow 0, \\
& 1-F(x) \sim\left\{1-\left[1-\exp \left\{-\alpha x^{\gamma} \exp (\lambda x)\right\}\right]^{a}\right\}^{b} \text { as } x \rightarrow \infty, \\
& f(x) \sim a b \alpha x^{\gamma-1}(\gamma+\lambda x) \exp \left\{\lambda x-\alpha x^{\gamma} \exp (\lambda x)\right\}\left[1-\exp \left\{-\alpha x^{\gamma} \exp (\lambda x)\right\}\right]^{a-1} \text { as } x \rightarrow 0, \\
& f(x) \sim a b \alpha x^{\gamma-1}(\gamma+\lambda x) \exp \left\{\lambda x-\alpha x^{\gamma} \exp (\lambda x)\right\}\left\{1-\left[1-\exp \left\{-\alpha x^{\gamma} \exp (\lambda x)\right\}\right]^{a}\right\}^{b-1} \text { as } x \rightarrow \infty,
\end{aligned}
$$




$$
\begin{aligned}
& h(x) \sim a b \alpha x^{\gamma-1}(\gamma+\lambda x) \exp \left\{\lambda x-\alpha x^{\gamma} \exp (\lambda x)\right\}\left[1-\exp \left\{-\alpha x^{\gamma} \exp (\lambda x)\right\}\right]^{a-1} \text { as } x \rightarrow 0, \\
& h(x) \sim a b \alpha x^{\gamma-1}(\gamma+\lambda x) \exp \left\{\lambda x-\alpha x^{\gamma} \exp (\lambda x)\right\}\left\{1-\left[1-\exp \left\{-\alpha x^{\gamma} \exp (\lambda x)\right\}\right]^{a}\right\}^{-1} \text { as } x \rightarrow \infty .
\end{aligned}
$$

\section{A useful expansion}

For $b>0$ real, Cordeiro et al. [4] demonstrated that the density function of $X$ can be expressed as

$$
f(x)=\sum_{s=0}^{\infty} b_{s} g_{s}(x)
$$

where the coefficient $b_{s}$ is given by

$$
b_{s}=\sum_{r=s}^{\infty} \frac{(-1)^{s}(r+1) d_{r}}{(s+1)}\left(\begin{array}{l}
r \\
s
\end{array}\right) \text {. }
$$

and

$$
g_{s}(x)=(s+1) \alpha x^{\gamma-1}(\gamma+\lambda x) \exp \left[\lambda x-\alpha(s+1) x^{\gamma} \exp (\lambda x)\right]
$$

denotes the density function of the $\mathrm{MW}((s+1) \alpha, \gamma, \lambda)$ distribution. Equation (3.1) reveals that the KwMW density function can be expressed as a linear combination of MW densities. Clearly, some mathematical properties of the KwMW distribution can be obtained directly from those properties of the MW distribution. If $b$ is an integer, the index $i$ in equation (3.1) stops at $b-1$.

\section{Quantile function}

Let $x=Q(u)$ be the quantile function of $X$. Cordeiro et al. [4] demonstrated that

$$
Q(u)=\sum_{i=1}^{\infty} a_{i}\left(-\alpha^{-1} \log \left\{1-\left[1-(1-u)^{1 / b}\right]^{1 / a}\right\}\right)^{i / \gamma},
$$

where

$$
a_{i}=\frac{(-1)^{i+1} i^{i-2}}{(i-1)}(\lambda / \gamma)^{i-1}
$$

A sample from the KwMW distribution may be obtained by applying its quantile function to a sample from a uniform distribution. Further, we can obtain the median, quantiles 25 and 75 by replacing $0.5,0.25$ and 0.75 in equation (4.1), respectively.

The shortcomings of the classical kurtosis measure are well-known; see Seier and Bonett [13] and Brys et al. [2]. There are many heavy-tailed distributions for which this measure is infinite, so it becomes uninformative precisely when it needs to be. Indeed, our motivation to use quantile-based measures stemmed from the non-existence of classical kurtosis for many distributions. The effect of the shape parameters $a$ and $b$ on the skewness and kurtosis of the KwMW distribution can be based on quantile measures. One of the earliest skewness measures to be suggested is the Bowley 

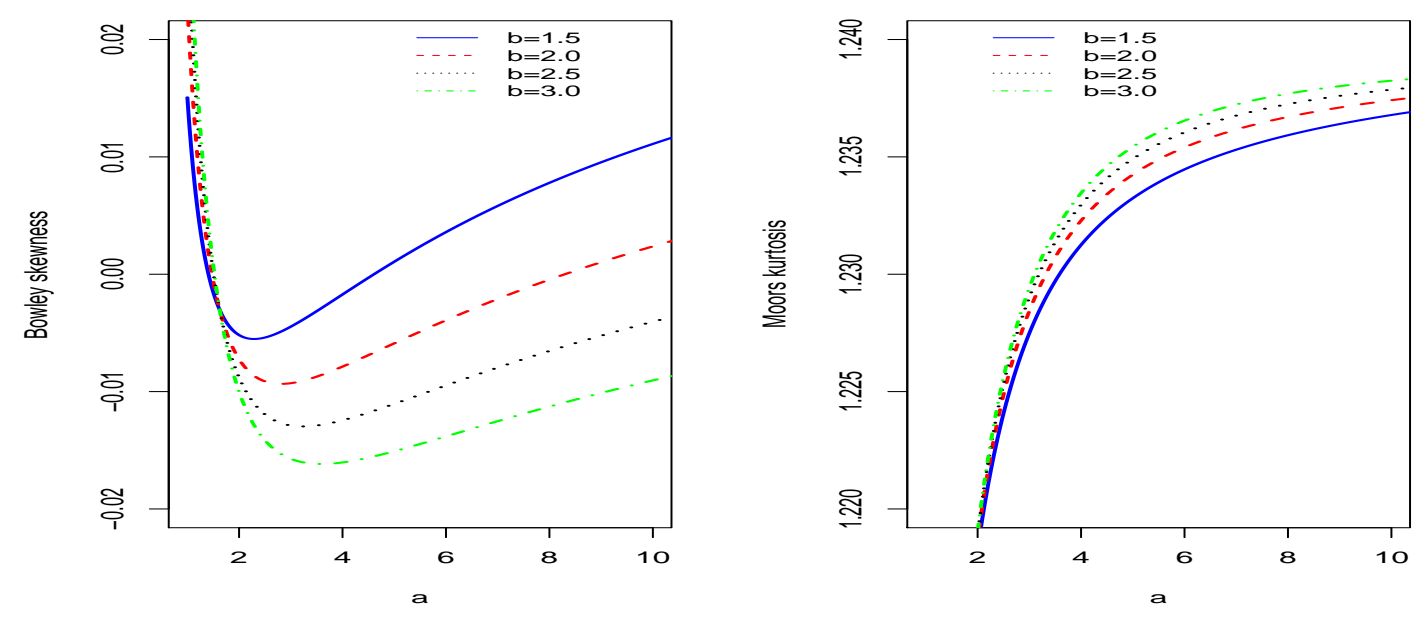

Fig. 2. Bowley skewness and Moors kurtosis of the KwMW distribution as a function of $a$ for some values of $b$.
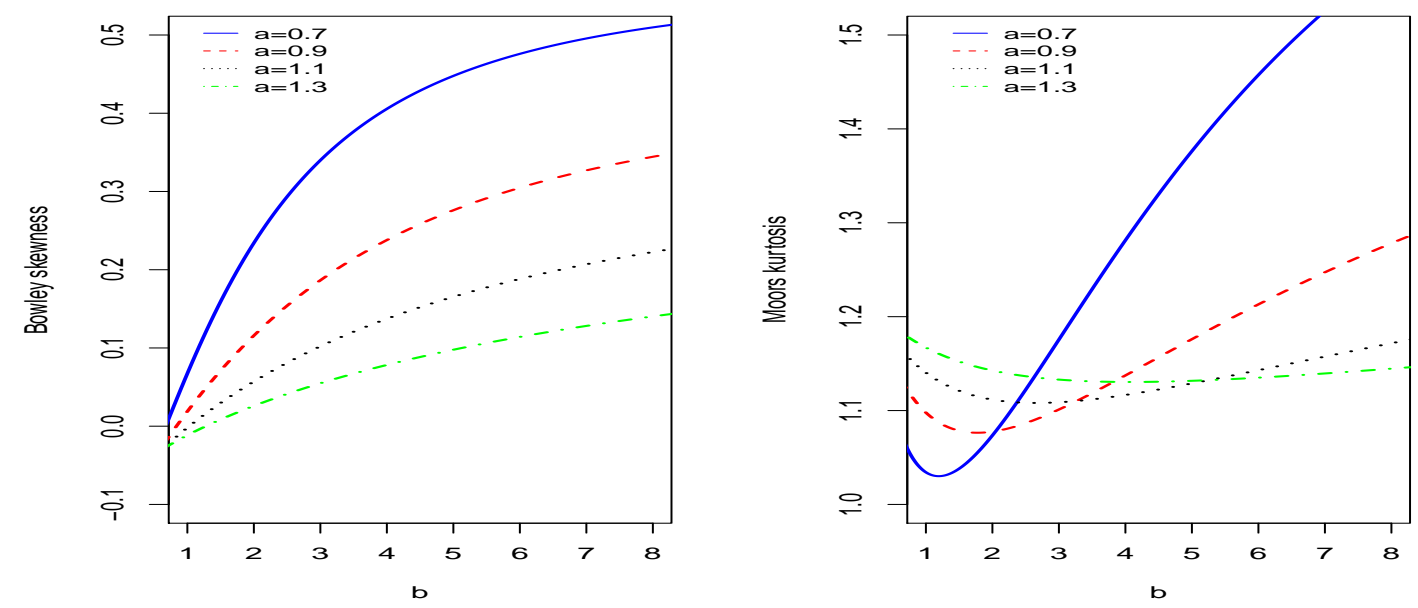

Fig. 3. Bowley skewness and Moors kurtosis of the KwMW distribution as a function of $b$ for some values of $a$.

skewness (Kenney and Keeping, [8]) defined by

$$
B=\frac{Q(3 / 4)+Q(1 / 4)-2 Q(1 / 2)}{Q(3 / 4)-Q(1 / 4)}
$$

On the other hand, the Moors kurtosis is based on octiles and given by

$$
M=\frac{Q(7 / 8)-Q(5 / 8)+Q(3 / 8)-Q(1 / 8)}{Q(6 / 8)-Q(2 / 8)} .
$$

The measures $B$ and $M$ are less sensitive to outliers and they exist even for distributions without moments. Figures 2 and 3 display the plots of the measures $B$ and $M$ as functions of $a$ and $b$ for some 
parameter values of the KwMW distribution, respectively. The plots indicate that these measures can be very sensitive on both shape parameters, thus indicating the importance of this distribution.

\section{Power series for the quantile function}

When the function $Q(u)$ does not have a closed-form expression, as is the case of the KwMW model, it can usually be written in terms of a power series. Here, we propose new explicit expressions for the moments and generating function of the KwMW distribution using a power series for the quantile function $x=Q(u)=F^{-1}(u)$ of $X$ obtained by inverting (1.3) with easily computed linear recurrence equation for its coefficients.

We consider an identity of Gradshteyn and Ryzhik ( [6], Section 0.314)) for a power series raised to any $j$ positive integer

$$
\left(\sum_{i=0}^{\infty} a_{i} x^{i}\right)^{j}=\sum_{i=0}^{\infty} c_{j, i} x^{i},
$$

where the coefficients $c_{j, i}$ (for $i=1,2, \ldots$ ) are easily obtained from the recurrence equation

$$
c_{j, i}=\left(i a_{0}\right)^{-1} \sum_{l=1}^{i}[l(j+1)-i] a_{l} c_{j, i-l}
$$

and $c_{j, 0}=a_{0}^{j}$. Equations (5.1) and (5.2) are used throughout this paper. The coefficients $c_{j, i}$ can be given explicitly in terms of the $a_{i}$ 's, although it is not necessary for programming numerically our expansions in any algebraic or numerical software.

Setting $t=x^{\gamma} \exp (\lambda x)$, we can express $x$ in terms of $t$ by (Cordeiro et al., [4])

$$
Q(u)=\sum_{i=1}^{\infty} a_{i} t^{i / \gamma}
$$

where the $a_{i}$ 's are given in the previous section. Considering this substitution and inverting (1.3), we can write

$$
t=-\alpha^{-1} \log \left\{1-\left[1-(1-u)^{1 / b}\right]^{1 / a}\right\} .
$$

By expanding (5.4), we obtain

$$
t=-\alpha^{-1} \log \left(1-\sum_{j=0}^{\infty} v_{j} u^{j}\right),
$$

where $v_{j}=\sum_{i=0}^{\infty}(-1)^{i+j}\left(\begin{array}{c}1 / a \\ i\end{array}\right)\left(\begin{array}{c}i / b \\ j\end{array}\right)$.

In Appendix A, based on equations (5.3) and (5.5) and other expansions, we demonstrate that the quantile function of $X$ admits a power series given by

$$
Q(u)=\sum_{j=0}^{\infty} h_{j} u^{j},
$$

where the basic quantities $c_{r, j}, p_{j}$ and $g_{r, j}$ are defined in Appendix A, $h_{j}=\sum_{i=1}^{\infty} \sum_{r=0}^{\infty} a_{i} g_{r, i} q_{r, j}$, $q_{r, j}=\left(j p_{0}\right)^{-1} \sum_{m=1}^{j}[m(r+1)-j] p_{m} c_{r, j-m}($ for $j \geq 1)$ and $c_{r, 0}=p_{0}^{r}$. 
Equation (5.6) is the main result of this section. We can derive from this equation alternative explicit expressions for the ordinary and incomplete moments, generating and characteristic functions and other statistical measures for the KwMW model.

\section{Moments}

\subsection{Ordinary, central and factorial moments}

The moments of the $X$ can be calculated from an infinite weighted linear combination of MW moments (Cordeiro et al., [4]). Here, we provide an alternative explicit expression for $\mu_{r}^{\prime}=E\left(X^{r}\right)$ based on the quantile power series. We can write from (5.6)

$$
\mu_{r}^{\prime}=\int_{0}^{\infty} x^{r} f(x) d x=\int_{0}^{1}\left(\sum_{j=0}^{\infty} h_{j} u^{j}\right)^{r} d u
$$

and then using (5.1) and (5.2), we obtain

$$
\mu_{r}^{\prime}=\sum_{j=0}^{\infty} \frac{v_{r, j}}{(j+1)}
$$

where the quantities $v_{r, j}$ (for $j=1,2, \ldots$ ) are easily determined from the recurrence equation

$$
v_{r, j}=\left(i h_{0}\right)^{-1} \sum_{m=1}^{j}[m(r+1)-j] h_{m} v_{r, j-m}
$$

and $v_{r, 0}=h_{0}^{r}$. We compare the numerical moments from equation (6.1) using a Mathematica script with those calculated by direct integration of the density function (1.4) for several choices of parameters. In all cases, the two methods are in good agreement by taking 20 terms in (6.1). Plots of the skewness and kurtosis when $\alpha=1.1, \gamma=0.9$ and $\lambda=0.2$ for some choices of $a$ as function of $b$ and for some choices of $b$ as function of $a$ are displayed in Figures 4 and 5, respectively. These plots indicate different behavior in terms of variation of the parameters $a$ and $b$ from those quantile moments presented in Section 4.

The central moments $\left(\mu_{r}\right)$ and cumulants $\left(\kappa_{r}\right)$ of $X$ can be determined from (6.1) as

$$
\mu_{r}=\sum_{k=0}^{r}(-1)^{k}\left(\begin{array}{l}
r \\
k
\end{array}\right) \mu_{1}^{\prime r} \mu_{r-k}^{\prime} \quad \text { and } \quad \kappa_{r}=\mu_{r}^{\prime}-\sum_{k=1}^{r-1}\left(\begin{array}{c}
r-1 \\
k-1
\end{array}\right) \kappa_{k} \mu_{r-k}^{\prime},
$$

respectively, where $\kappa_{1}=\mu_{1}^{\prime}$. Thus, $\kappa_{2}=\mu_{2}^{\prime}-\mu_{1}^{\prime 2}, \kappa_{3}=\mu_{3}^{\prime}-3 \mu_{2}^{\prime} \mu_{1}^{\prime}+2 \mu_{1}^{\prime 3}, \kappa_{4}=\mu_{4}^{\prime}-4 \mu_{3}^{\prime} \mu_{1}^{\prime}-$ $3 \mu_{2}^{\prime 2}+12 \mu_{2}^{\prime} \mu_{1}^{\prime 2}-6 \mu_{1}^{\prime 4}$, etc. The skewness $\gamma_{1}=\kappa_{3} / \kappa_{2}^{3 / 2}$ and kurtosis $\gamma_{2}=\kappa_{4} / \kappa_{2}^{2}$ can be calculated from the third and fourth standardized cumulants. The $r$ th descending factorial moment of $X$ is

$$
\mu_{(r)}^{\prime}=E\left[X^{(r)}\right]=E[X(X-1) \times \cdots \times(X-r+1)]=\sum_{n=0}^{r} s(r, n) \mu_{n}^{\prime},
$$

where $s(r, n)=(n !)^{-1}\left[d^{n} x^{(r)} / d x^{n}\right]_{x=0}$ is the Stirling number of the first kind.

\subsection{Incomplete moments}

The $r$ th incomplete moment of $X$ is defined by $m_{r}(y)=E\left(X^{r} \mid X<y\right)=\int_{0}^{y} x^{r} f(x) d x$. Here, we propose two methods to calculate the KwMW incomplete moments. The first method is based on 

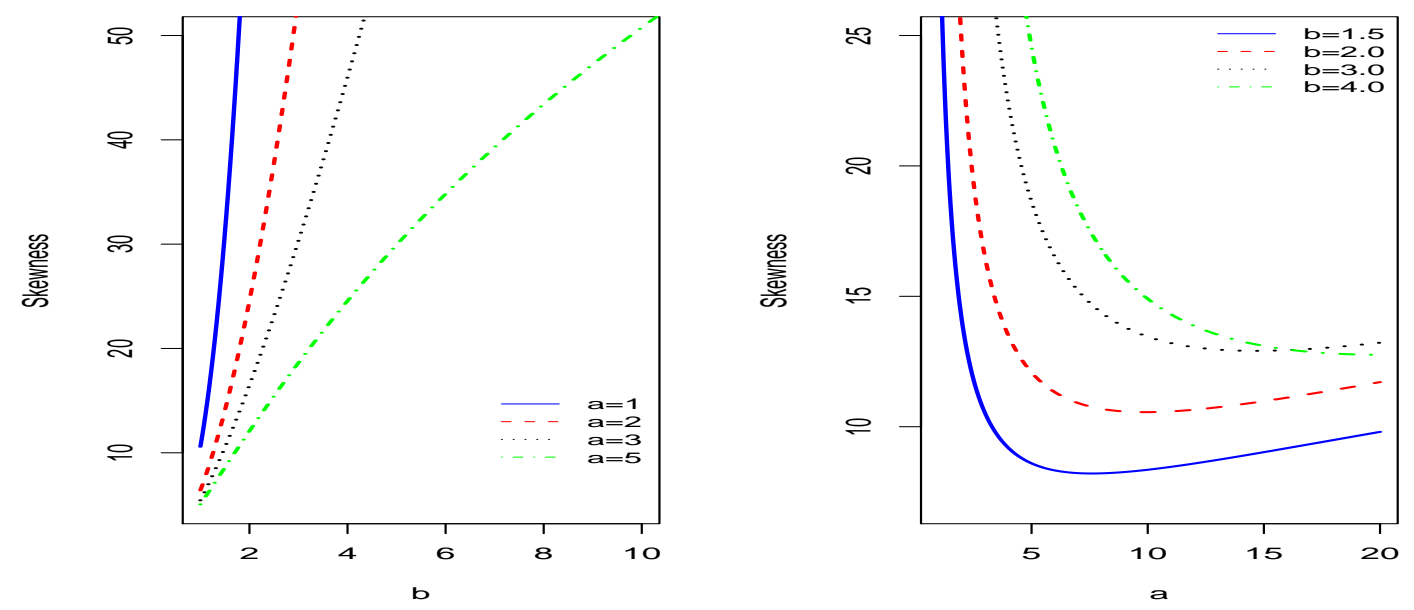

Fig. 4. KwMW skewness as functions of $b$ and $a$ for fixed values of the other parameter.
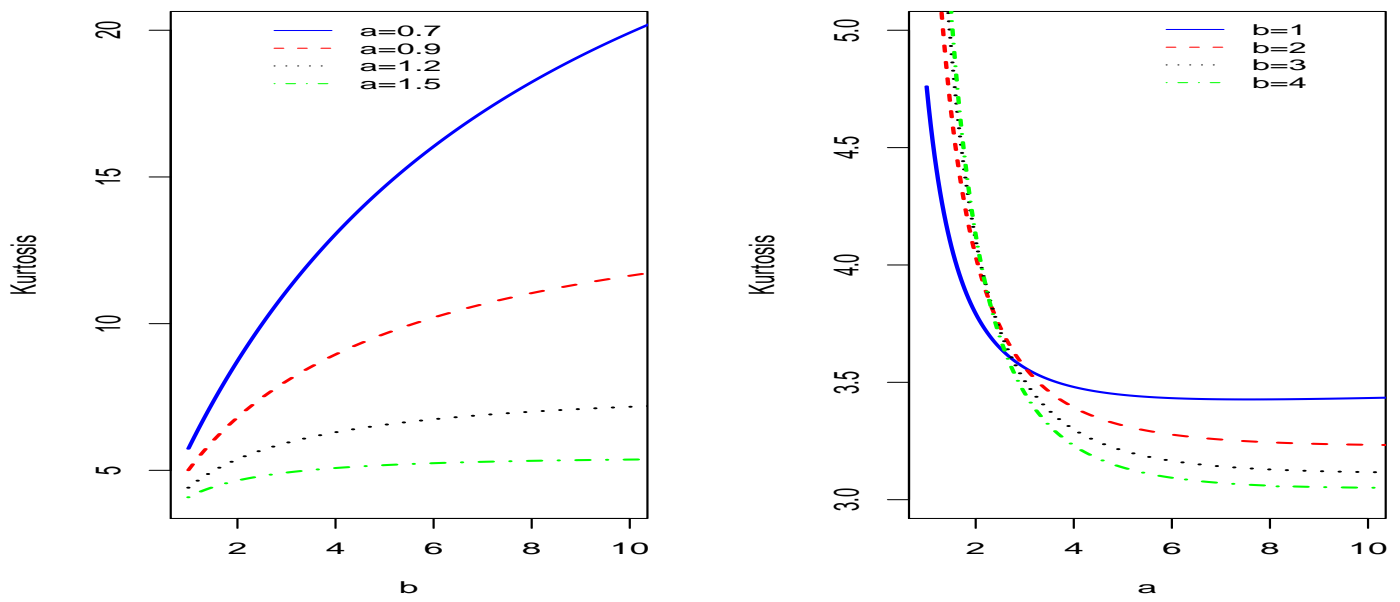

Fig. 5. KwMW kurtosis as functions of $b$ and $a$ for fixed values of the other parameter.

the quantile power series. We can write from (5.6)

$$
\mu_{r}(y)=\int_{0}^{y} x^{r} f(x) d x=\int_{0}^{F(y)}\left(\sum_{j=0}^{\infty} h_{j} u^{j}\right)^{r} d u,
$$

and using similar algebra for the ordinary moments, we obtain

$$
\mu_{r}(y)=\sum_{j=0}^{\infty} \frac{v_{r, j} F(y)^{j+1}}{(j+1)},
$$

where $F(y)$ is given by (1.3). 
The second method requires more algebra and follows from the linear combination (3.1)

$$
m_{r}(y)=\sum_{j=0}^{\infty} b_{j} t_{r}(j)
$$

where $t_{r}(j)=\int_{0}^{y} x^{r} g_{j}(x) d(x)$ denotes the $r$ th MW incomplete moment under the parameters $(j+$ 1) $\alpha, \gamma$ and $\lambda$ given by

$$
t_{r}(j)=(j+1) \alpha \int_{0}^{y} x^{r+\gamma-1}(\gamma+\lambda x) \exp (\lambda x) \exp \left\{-(j+1) \alpha x^{\gamma} \exp (\lambda x)\right\} d x .
$$

Let $z=x^{\gamma} \exp (\lambda x)$. We can invert this equation to obtain $x$ in terms of $z$ (when both $\lambda$ and $\gamma$ are positive) as

$$
x=\frac{\gamma}{\lambda} F\left(\frac{\lambda z^{1 / \gamma}}{\gamma}\right)
$$

where

$$
F(w)=\sum_{k=1}^{\infty} \frac{(-1)^{k+1} k^{k-2} w^{k}}{(k-1) !} .
$$

Further, we can express $x$ in terms of $z$ from equation (6.5) by

$$
x=\sum_{k=1}^{\infty} p_{k} z^{k / \gamma},
$$

where $p_{k}=\gamma \lambda^{k} a_{k} /\left(\lambda \gamma^{k}\right)$ and $a_{k}$ were defined in Section 5 .

Changing the variable $x$ by $z$, the integral in (6.4) becomes

$$
I_{j}=\int_{0}^{y^{\gamma} \exp (\lambda y)}\left(\sum_{k=1}^{\infty} p_{k} z^{k / \gamma}\right)^{r} \exp \{-(j+1) \alpha z\} d z .
$$

We can write

$$
\left(\sum_{k=1}^{\infty} p_{k} z^{k / \gamma}\right)^{r}=\sum_{k_{1}, \ldots, k_{r}=1}^{\infty} A_{k_{1}, \ldots, k_{r}} z^{s_{r} / \gamma}
$$

where $A_{k_{1}, \ldots, k_{r}}=a_{k_{1}} \ldots a_{k_{r}}$ and $s_{r}=k_{1}+\ldots+k_{r}$. So, the quantity $I_{j}$ can be rewritten as

$$
I_{j}=\sum_{k_{1}, \ldots, k_{r}=1}^{\infty} A_{k_{1}, \ldots, k_{r}} \int_{0}^{y^{\gamma} \exp (\lambda y)} z^{s_{r} / \gamma} \exp \{-(j+1) \alpha z\} d z
$$

Setting $t=(j+1) \alpha z$, we can obtain $I_{j}$ and then, equation (6.4) becomes

$$
t_{r}(j)=(j+1) \alpha \sum_{k_{1}, \ldots, k_{r}=1}^{\infty} \frac{A_{k_{1}, \ldots, k_{r}} \gamma\left(k / \gamma+1,(j+1) \alpha y^{\left.\gamma \mathrm{e}^{\lambda \mathrm{y}}\right)}\right.}{\{(\mathrm{j}+1) \alpha\}^{\mathrm{s}_{\mathrm{r}} / \gamma+1}} .
$$

Substituting the last equation in (6.3), we obtain

$$
m_{r}(y)=\alpha \sum_{j=0}^{\infty} \sum_{k_{1}, \ldots, k_{r}=1}^{\infty}(j+1) b_{j} \frac{A_{k_{1}, \ldots, k_{r}} \gamma\left(s_{r} / \gamma+1,(j+1) \alpha y^{\gamma} e^{\lambda y}\right)}{\{(j+1) \alpha\}^{s_{r} / \gamma+1}}
$$


Incomplete moments can be used to obtain Bonferroni and Lorenz curves which are applied in economics, reliability, demography, insurance and medicine. For the random variable $X$, they are defined by $B(p)=m_{1}(q) / \mu$ and $L(p)=m_{1}(q) / \mu$, respectively, where $\mu_{1}^{\prime}=E(X)$ and $q=F^{-1}(p)$ is the quantile function of $X$. Thus, using (6.6), these curves can be expressed as

$$
B(p)=\frac{\alpha}{p \mu_{1}^{\prime}} \sum_{j=0}^{\infty} \sum_{k_{1}, \ldots, k_{r}=1}^{\infty}(j+1) b_{j} \frac{A_{k_{1}, \ldots, k_{r}} \gamma\left(s_{1} / \gamma+1,(j+1) \alpha q^{\gamma} e^{\lambda q}\right)}{[(j+1) \alpha]^{s_{1} / \gamma+1}}
$$

and $L(p)=B(p) / \mu_{1}^{\prime}$, respectively. The Lorenz curve is displayed in Figure 6 for $a=$ $6,10,20,50,100$ and $b=1.1, \alpha=1.1, \gamma=1.1$ and $\lambda=0.01$. The Bonferroni curve is displayed in Figure 7 for $a=5,10,20,30,50$ and $b=2.0, \alpha=0.5, \gamma=0.5$ and $\lambda=0.1$. The area between the Bonferroni curve and $B(p)=1$ is known as the Bonferroni concentration index, $B(X)$, and is given by

$$
B(X)=\int_{0}^{1}[1-B(p)] d p=1-\int_{0}^{1} B(p) d p .
$$

The area between the Lorenz curve and $L(p)=p$ is known as the area of concentration. The Gini concentration index, $C(X)$, is twice this area given by

$$
C(X)=2 \int_{0}^{1}[p-L(p)] d p=1-2 \int_{0}^{1} L(p) d p .
$$

(a)

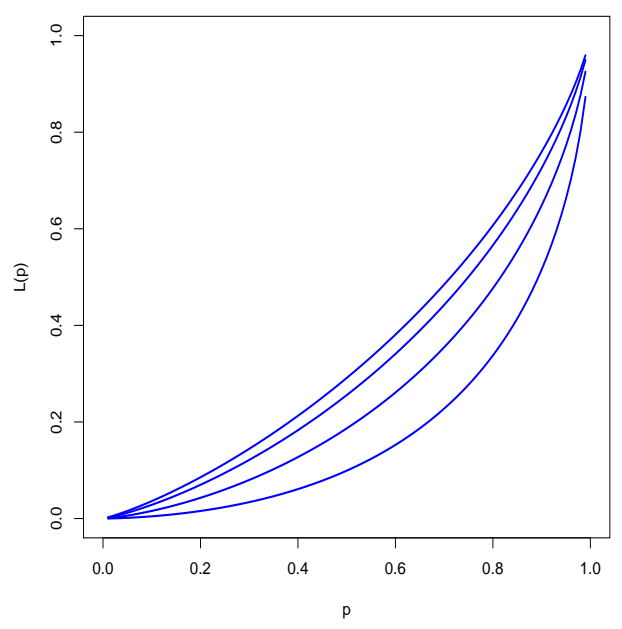

(b)

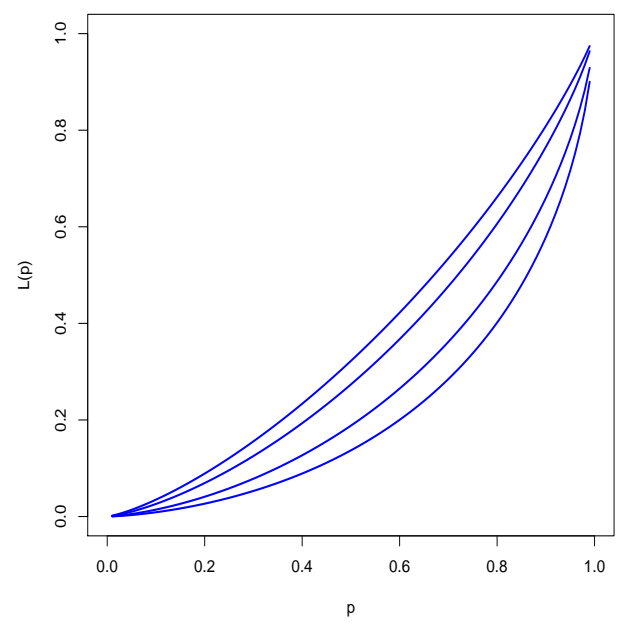

Fig. 6. The KwMW Lorenz curve. (a) For $a=10,40,150,350$ and $b=1.1, \alpha=1.1, \gamma=0.3$ and $\lambda=0.0001$. The curves from the bottom to top correspond to increasing values of $a$. (b) For $a=20$ and $b=1,2,10,60, \alpha=1.1, \gamma=0.3$ and $\lambda=0.0001$. The curves from the bottom to top correspond to increasing values of $b$.

Equations (6.1), (6.2), (6.3) and (6.6) are the main results of this section. 
(a)

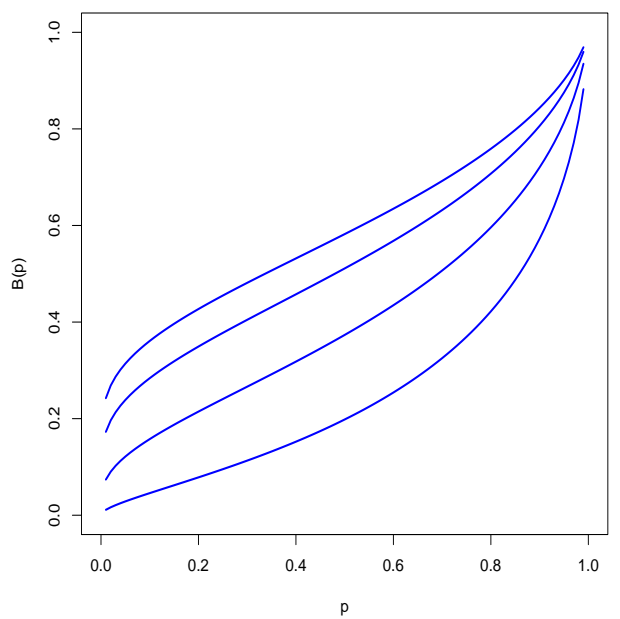

(b)

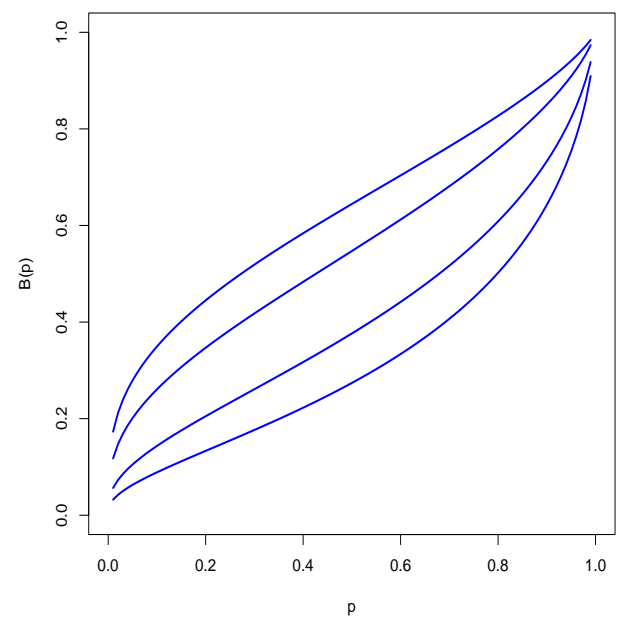

Fig. 7. The KwMW Bonferroni curve. (a) For $a=10,40,150,350$ and $b=1.1, \alpha=1.1, \gamma=0.3$ and $\lambda=0.0001$. The curves from the bottom to top correspond to increasing values of $a$. (b) For $a=20$ and $b=1,2,10,60, \alpha=1.1, \gamma=0.3$ and $\lambda=0.0001$. The curves from the bottom to top correspond to increasing values of $b$.

\section{Generating function}

Cordeiro et al. [4] provided a representation for the moment generating function (mgf) of $X$, $M(t)=E\left(\mathrm{e}^{\mathrm{tX}}\right)$ say, computed from equation (3.1) as an infinite weighted linear combination of MW generating functions. They obtained

$$
M(t)=\sum_{s, n=0}^{\infty} \frac{b_{s} t \gamma(-1)^{n+1}(n \lambda-t \gamma)^{n-1}}{n ! \gamma^{n}\{(s+1) \alpha\}^{n / \gamma}} \Gamma\left(\frac{n}{\gamma}+1\right) .
$$

We now provide a new alternative representation for $M(t)$ in (7.1) based on the quantile power series (5.6). We can write

$$
M(t)=\int_{0}^{\infty} \mathrm{e}^{\mathrm{tx}} \mathrm{f}(\mathrm{x}) \mathrm{dx}=\int_{0}^{1} \exp \left[\mathrm{t}\left(\sum_{\mathrm{j}=0}^{\infty} \mathrm{h}_{\mathrm{j}} \mathrm{u}^{\mathrm{j}}\right)\right] \mathrm{du} .
$$

We expand the exponential function and use the same algebra that leads to (6.1)

$$
M(t)=\int_{0}^{1} \sum_{r=0}^{\infty} \frac{t^{r}\left(\sum_{j=0}^{\infty} h_{j} u^{j}\right)^{r}}{r !} d u=\int_{0}^{1} \sum_{r=0}^{\infty} \frac{t^{r} v_{r, j} u^{j}}{r !} d u
$$

and then

$$
M(t)=\sum_{r, j=0}^{\infty} \frac{t^{r} v_{r, j}}{(j+1) r !} .
$$

Equation (7.2) is a good alternative in terms of computing time to formula (7.1). 


\section{A KwMW mixture model with cure fraction}

In population based cancer studies, cure is said to occur when the mortality in the group of cancer patients returns to the same level as that expected in the general population. The cure fraction is of interest to patients and also a useful measure when analyzing trends in cancer patient survival. Cure rate models have been used for modeling time-to-event data for various types of cancers, including breast cancer, non-Hodgkins lymphoma, leukemia, prostate cancer and melanoma. Perhaps the most popular type of cure rate models is the mixture model (Berkson and Gage, [1]; Maller and Zhou, [10]). In this model, the population is divided into two sub-populations so that an individual either is cured with probability $\pi$, or has a proper survival function $S(x)$ with probability $1-\pi$. This formulation leads to an improper population survivor function $S^{*}(x)$ expressed in the mixture form

$$
S^{*}(x)=\pi+(1-\pi) S(x), S(\infty)=0, S^{*}(\infty)=\pi
$$

Common choices for $S(x)$ in (8.1) are the exponential and Weibull distributions. Here, we adopt the KwMW distribution. Mixture models involving these distributions have been studied by several authors, including Farewell [5], Sy and Taylor [14] and Ortega et al. [12]. The use of survival models with a cure fraction has become more and more frequent because traditional survival analysis do not allow for modeling data in which nonhomogeneous parts of the population do not represent the event of interest even after a long follow-up. Now, we propose an application of the new distribution to compose a mixture model for cure rate estimation.

Suppose that the $X_{i}$ 's are independent and identically distributed random variables having the density function (1.4). Consider a sample $x_{1}, \ldots, x_{n}$, where $x_{i}$ is either the observed lifetime or censoring time for the $i$ th individual. Let a binary random variable $z_{i}$ (for $i=1, \ldots, n$ ) indicate that the $i$ th individual in a population is at risk or not with respect to a certain type of failure, i.e. $z_{i}=1$ indicates that the $i$ th individual will eventually experience a failure event (uncured) and $z_{i}=0$ indicates that the individual will never experience such event (cured). The proportion of uncured $1-\pi$ individuals can be expressed such that the conditional distribution of $z_{i}$ is $\operatorname{Pr}\left(z_{i}=1\right)=1-\pi$.

The maximum likelihood method is used to estimate the model parameters. So, the contribution of an individual that failed at $x_{i}$ to the likelihood function reduces to

$$
\begin{aligned}
& (1-\pi) a b \alpha(\gamma+\lambda x) x^{\gamma-1} \exp \left[\lambda x-\alpha x^{\gamma} \exp (\lambda x)\right] \\
& \times\left[1-\exp \left\{-\alpha x^{\gamma} \exp (\lambda x)\right\}\right]^{a-1}\left\{1-\left[1-\exp \left\{-\alpha x^{\gamma} \exp (\lambda x)\right\}\right]^{a}\right\}^{b-1}
\end{aligned}
$$

whereas the contribution of an individual that is at risk at time $x_{i}$ becomes

$$
\pi+(1-\pi)\left\{1-\left[1-\exp \left\{-\alpha x^{\gamma} \exp (\lambda x)\right\}\right]^{a}\right\}^{b} .
$$

The new model defined by (8.2) and (8.3) is called the KwMW mixture model with long-term survivors. For $a=b=1$, we obtain a special model called the MW mixture model with long-term survivors. 
Thus, the log-likelihood function for the parameter vector $\theta=(a, b, \alpha, \gamma, \lambda)^{T}$ can be obtained from equations (8.2) and (8.3) as

$$
\begin{aligned}
l(\lambda)= & r \log [(1-\pi) a b \alpha]+(\gamma-1) \sum_{i \in F} \log \left(\gamma+\lambda x_{i}\right)+\sum_{i \in F} \log \left[\lambda x_{i}+\log \left(u_{i}\right)\right] \\
& +(a-1) \sum_{i \in F} \log \left(1-u_{i}\right)+(b-1) \sum_{i \in F} \log \left[1-\left(1-u_{i}\right)^{a}\right] \\
& +\sum_{i \in C} \log \left\{\pi+(1-\pi)\left[1-\left(1-u_{i}\right)^{a}\right]^{b}\right\}
\end{aligned}
$$

where $u_{i}=\exp \left[-\alpha x_{i}^{\gamma} \exp \left(\lambda x_{i}\right)\right], F$ and $C$ denote the sets of individuals corresponding to lifetime observations and censoring times, respectively, and $r$ is the number of uncensored observations (failures).

\section{Simulation results}

We shall report the results from a Monte Carlo experiment on the finite sample behavior of the MLEs of the parameters $a, b, \alpha, \gamma$ and $\lambda$. The simulation was carried out using the $\mathrm{R}$ programming language and were obtained from 10,000 Monte Carlo replications. In each replication, a random sample of size $n$ is drawn from the $\operatorname{KwMW}(a, b, \alpha, \gamma, \lambda)$ distribution and the parameters were estimated by maximum likelihood. In Table 1, we present the means of the MLEs of the five parameters with the corresponding root mean squared error (RMSE) for sample sizes 50, 100, 300 and 500. The true parameters values used in the data generating processes are $a=10.5, b=2.5, \alpha=3.5, \gamma=2.5$ and $\lambda=2.5$. The figures in Table 1 indicate that the bias and RMSE of the estimates approach zero

Table 1. Mean estimates and root mean squared errors of the MLEs based Monte Carlo simulation.

\begin{tabular}{c|cccc|cccc}
\hline Parameter & \multicolumn{5}{|c|}{ Mean } & \multicolumn{4}{c}{ RMSE } \\
\hline & $\mathrm{n}=50$ & $\mathrm{n}=100$ & $\mathrm{n}=300$ & $\mathrm{n}=500$ & $\mathrm{n}=50$ & $\mathrm{n}=100$ & $\mathrm{n}=300$ & $\mathrm{n}=500$ \\
\hline$a$ & 11.6704 & 10.8372 & 10.6686 & 10.4484 & 9.0340 & 6.4256 & 4.9804 & 3.9874 \\
$b$ & 4.6927 & 3.7767 & 3.2871 & 2.8526 & 6.0056 & 4.3980 & 3.2566 & 2.3479 \\
$\alpha$ & 4.3568 & 4.0180 & 3.9600 & 3.8154 & 3.1482 & 2.5704 & 2.2652 & 1.5560 \\
$\gamma$ & 4.1065 & 3.2403 & 2.9083 & 2.8120 & 3.7580 & 1.6572 & 0.8231 & 0.7360 \\
$\lambda$ & 4.4326 & 3.6422 & 3.1789 & 3.0286 & 4.1014 & 2.6645 & 2.1311 & 1.8689 \\
\hline
\end{tabular}

as the sample size increases. Figures 8 and 9 display the histogram of the MLEs of the five parameters for sample sizes 50 and 500. We note that the dispersion for all estimates of the parameters decreases when the sample size increases as expected.

\section{Application: Melanoma data set with long-term survivors}

In this section, the proposed model for survival data with cure fraction is applied to a real data set on cancer recurrence. The data are part of a study on cutaneous melanoma (a type of malignant cancer) for the evaluation of postoperative treatment performance with a high dose of a certain drug (interferon alfa-2b) in order to prevent recurrence. Patients were included in the study from 1991 to 1995 and follow-up was conducted until 1998. The data were collected by Ibrahim et al. [7]. The 
(a)

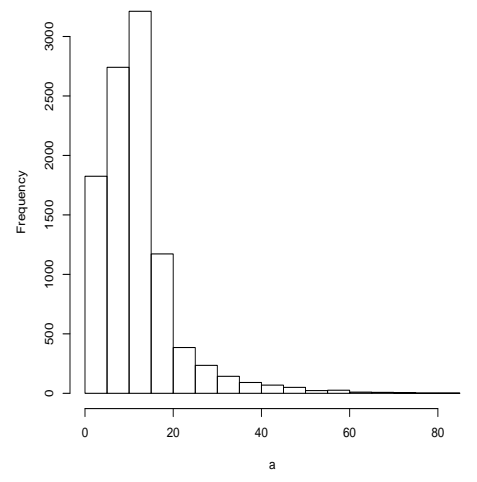

(b)

(c)

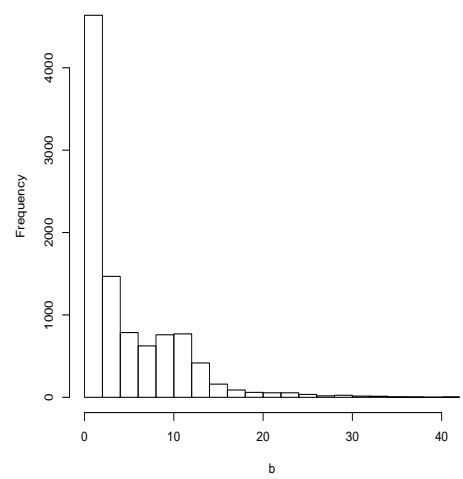

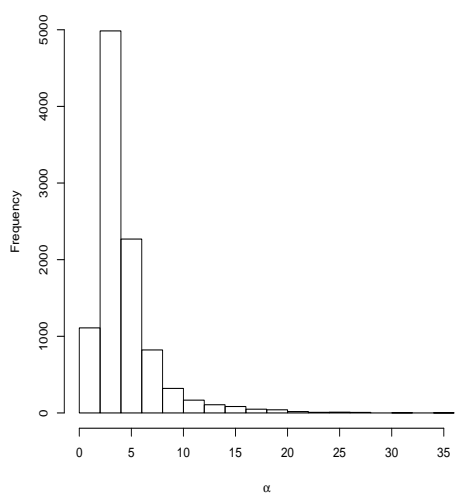

(d)

(e)
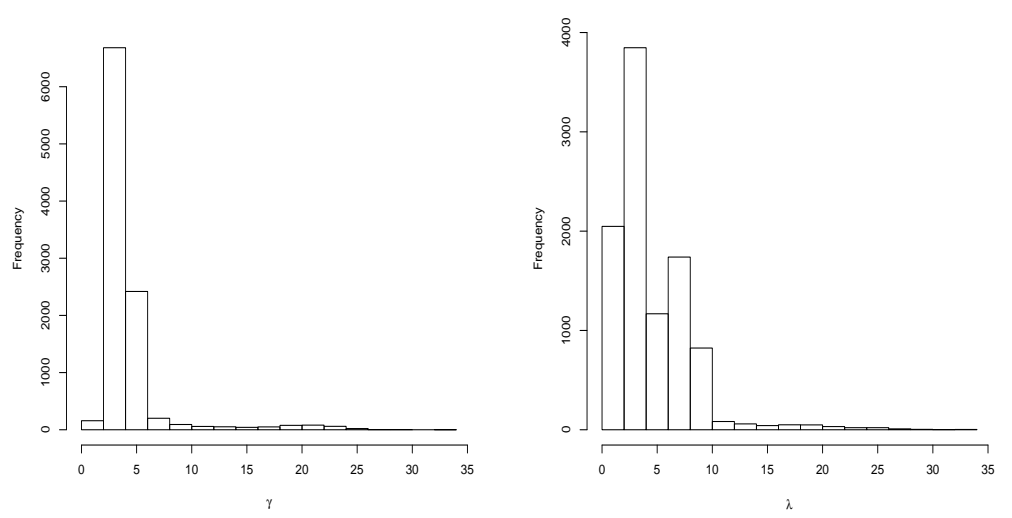

Fig. 8. Histograms for the 10,000 simulated values of the MLE based in $n=50$. (a) For $a$. (b) For $b$. (c) For $\alpha$. (d) For $\gamma$. (e) For $\lambda$

survival time $X$ is defined as the time until the patient's death. The original sample size was $n=427$ patients, 10 of whom did not present a value for the explanatory variable tumor thickness. When such cases were removed, we obtain a sample of $n=417$ patients. The percentage of censored observations was $56 \%$. 
(a)

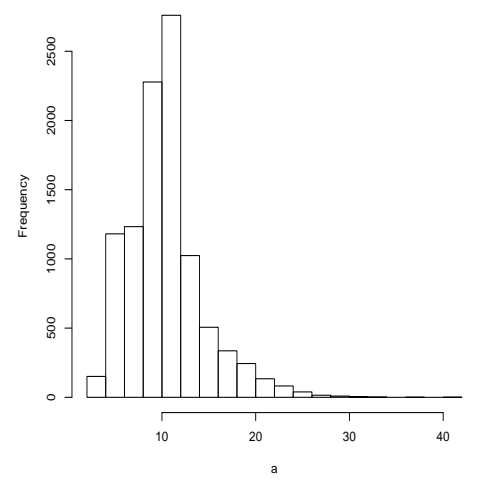

(b)

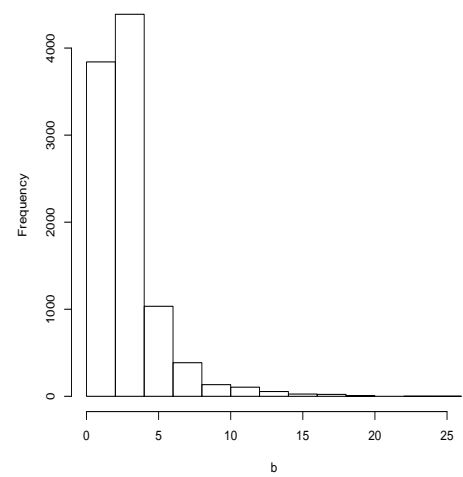

(c)

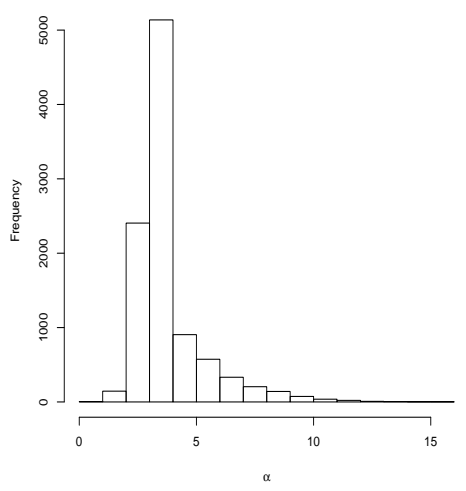

(d)

(e)
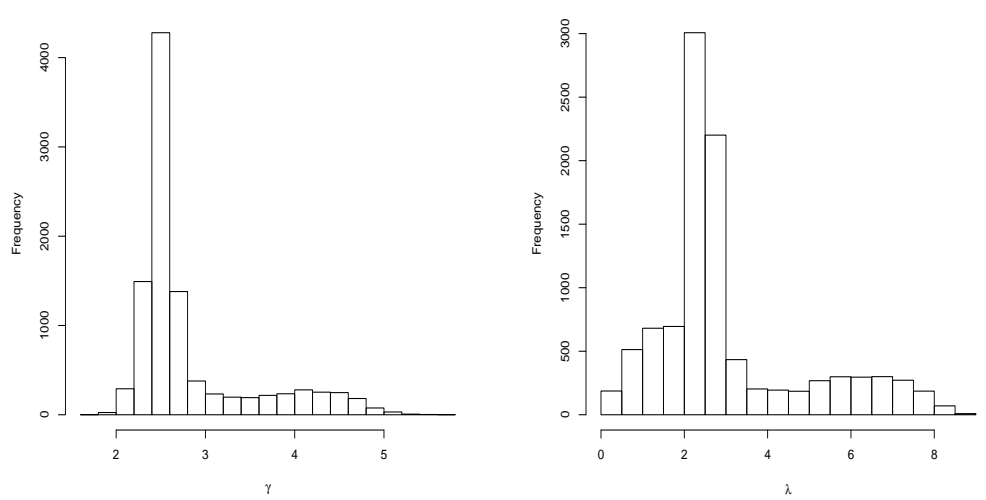

Fig. 9. Histograms for the 10,000 simulated values of the MLE based in $n=500$. (a) For $a$. (b) For $b$. (c) For $\alpha$. (d) For $\gamma$. (e) For $\lambda$.

In order to estimate the model parameters, we adopt the maximum likelihood method.We take the estimates of $\alpha, \gamma$ and $\lambda$ from the fitted MW mixture model as starting values for the numerical iterative procedure. The computations were performed using the statistical software R. Table 2 lists the MLEs of the model parameters and the values of the Akaike information criterion (AIC). 
The results indicate that the KwMW mixture model has the smaller value of this statistic when compared to that value of the MW mixture model. The LR statistic for testing the hypotheses $H_{0}: a=$ $b=1$ versus $H_{1}: H_{0}$ is not true, i.e. to compare the KwMW and MW mixture models, becomes $w=2\{-524.64-(-529.37)\}=9.46(p$-value $=0.008)$, which indicates that the KwMW mixture model is superior to the MW mixture model in terms of model fitting. Figure 10 displays plots of the empirical survival function and the estimated survival function for the KwMW mixture. Note that the proportion of cured estimated by the KwMW mixture model $\left(\hat{\pi}_{K w M W}=0.4887\right)$ is more appropriate than the MW mixture model $\left(\hat{\pi}_{M W}=0.5060\right)$. Further, the KwMW mixture model provides a better fit to these data.

Table 2. MLEs of the model parameters for the melanoma data, the corresponding SEs (given in parentheses) and the AIC statistic.

\begin{tabular}{c|cccccc|c}
\hline Model & $\pi$ & $a$ & $b$ & $\alpha$ & $\gamma$ & $\lambda$ & AIC \\
\hline KwMW Mixture & 0.4887 & 5.6169 & 1.3866 & 1.3103 & 0.6102 & 0.0003 & 1061.2 \\
& $(0.0349)$ & $(0.2949)$ & $(0.0801)$ & $(0.0763)$ & $(0.0429)$ & $(0.00015)$ & \\
\hline MW Mixture & 0.5060 & $(-)$ & $(-)$ & 0.2401 & 1.7369 & 0.0006 & 1066.7 \\
& $(0.0313)$ & $(-)$ & $(-)$ & $(0.0324)$ & $(0.0986)$ & $(0.0003)$ & \\
\hline
\end{tabular}

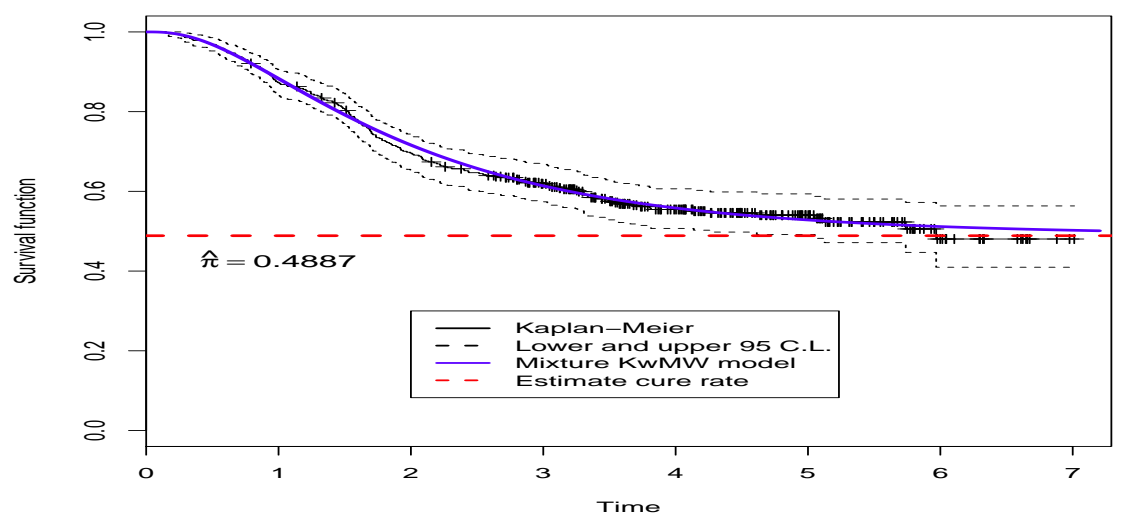

Fig. 10. Estimated survival function for the KwMW mixture model and the empirical survival for melanoma data.

\section{Bivariate generalization}

The KwMW distribution can be easily generalized to the bivariate and multivariate cases. Consider the bivariate case for simplicity. Let $G\left(x_{1}, x_{2}\right)$ denote a bivariate cdf on $(0, \infty) \times(0, \infty)$ with joint pdf $g\left(x_{1}, x_{2}\right)$, marginal pdfs $g_{i}\left(x_{i}\right), i=1,2$ and marginal cdfs $G_{i}\left(x_{i}\right)$ for $i=1,2$. For $a>0$ and $b>0$, we define the cdf of the nine-parameter bivariate KwMW distribution as $F\left(x_{1}, x_{2}\right)=1-[1-$ 
$\left.G^{a}\left(x_{1}, x_{2}\right)\right]^{b}$, where $G\left(x_{1}, x_{2}\right)$ is the seven-parameter bivariate MW distribution given by

$$
\begin{aligned}
G\left(x_{1}, x_{2}\right)= & 1+\exp \left\{-\left[\left(\alpha_{1} x_{1}^{\gamma_{1}} \exp \left(\lambda_{1} x_{1}\right)\right)^{\frac{1}{\delta}}+\left(\alpha_{2} x_{2}^{\gamma_{2}} \exp \left(\lambda_{2} x_{2}\right)\right)^{\frac{1}{\delta}}\right]^{\delta}\right\} \\
& -\exp \left[-\alpha_{1} x_{1}^{\gamma_{1}} \exp \left(\lambda_{1} x_{1}\right)\right]-\exp \left[-\alpha_{2} x_{2}^{\gamma_{2}} \exp \left(\lambda_{2} x_{2}\right)\right],
\end{aligned}
$$

for $\alpha_{i}>0, \gamma_{i}>0$ and $\lambda_{i} \geq 0$ for $i=1,2$ and the parameter $0<\delta \leq 1$ measures the association between $X_{1}$ and $X_{2}$. If these random variables are independent then $\delta=0$. The bivariate MW density function can be expressed as

$$
\begin{aligned}
g\left(x_{1}, x_{2}\right)= & -\frac{1}{\delta x_{1} x_{2}}\left\{\left(\gamma_{1}+q_{1}\right)\left(\gamma_{2}+q_{2}\right) \exp \left(-q_{1}-q_{2}\right)\left(p_{1}\right)^{\frac{1}{\delta}}\left(p_{2}\right)^{\frac{1}{\delta}} \times\right. \\
& \exp \left\{q_{2}-\left[\left(p_{1}\right)^{\frac{1}{\delta}}+\left(p_{2}\right)^{\frac{1}{\delta}}\right]^{\delta}+q_{1}\right\}\left\{(\delta-1)\left[\left(p_{1}\right)^{\frac{1}{\delta}}+\left(p_{2}\right)^{\frac{1}{\delta}}\right]^{\delta-2}\right. \\
& \left.-\delta\left[\left(p_{1}\right)^{\frac{1}{\delta}}+\left(p_{2}\right)^{\frac{1}{\delta}}\right]^{2 \delta-2}\right\},
\end{aligned}
$$

where $p_{i}=\alpha_{i} x_{i}^{\gamma_{i}} \mathrm{e}^{\mathrm{q}_{\mathrm{i}}}$ and $q_{i}=\lambda_{i} x_{i}$. The marginal pdf's $g_{i}(x)$ and cdf's $G_{i}(x)$ (for $\left.i=1,2\right)$ are given by $g_{i}\left(x_{i}\right)=\alpha_{i} x_{i}^{\gamma_{i}-1}\left(\gamma_{i}+\lambda_{i} x_{i}\right) \mathrm{e}^{\lambda_{\mathrm{i}} \mathrm{x}_{\mathrm{i}}} \exp \left\{\lambda_{\mathrm{i}} \mathrm{x}_{\mathrm{i}}-\alpha_{\mathrm{i}} \mathrm{x}_{\mathrm{i}}^{\gamma_{i}}\right\}$ and $G_{i}\left(x_{i}\right)=1-\exp \left\{-\alpha_{i} x_{i}^{\gamma_{i}} \exp \left(\lambda_{i} x_{i}\right)\right\}$. The marginal pdf's $f_{i}(x)$ and cdf's $F_{i}(x)$ (for $\left.i=1,2\right)$ are $f_{i}(x)=a b g_{i}(x) G_{i}(x)^{a-1}\left[1-G_{i}(x)^{a}\right]^{b-1}$ and $F_{i}(x)=1-\left[1-G_{i}(x)^{a}\right]^{b}$, where $g_{i}(x)$ and $G_{i}(x)$ are given before.

We hope to provide a comprehensive mathematical treatment of the bivariate and multivariate KwMW distribution in a future paper.

\section{Conclusions}

In this paper, we study some new mathematical properties not investigated by Cordeiro et al. [4] of the Kumaraswamy modified Weibull (KwMW) distribution. This broader class of distributions is quite flexible in analyzing positive data and includes several special models discussed in the literature such as the Kumaraswamy Weibull, generalized modified Weibull, exponentiated Weibull, exponentiated exponential, Kumaraswamy modified exponential, generalized modified Rayleigh, generalized modified exponential, Rayleigh and Weibull distributions (see Figure 1). We derive a power series expansion for the KwMW quantile function. We provide new explicit expressions for the ordinary and incomplete moments, quantile moments, mean deviations, Bonferroni and Lorenz curves and generating function. Our formulas related with the KwMW are manageable, and with the use of modern computer resources with analytic and numerical capabilities, may turn into adequate tools comprising the arsenal of applied statisticians. Simulation results were performed to show the consistence of the new distribution. We define a KwMW mixture model for survival data with longterm survivors. The practical relevance and applicability of the current models are demonstrated by means of one real data set. Finally, we also propose a new bivariate KwMW distribution.

\section{Acknowledgments}

The authors are grateful to the referees and the Editor for very useful comments and suggestions. This work was supported by CNPq (Brazil) and CAPES (Brazil). 


\section{Appendix A. Quantile function}

Using the logarithmic expansion for $z \in(0,1)$ in (5.5) $\log (1-z)-z \sum_{k=0}^{\infty} \frac{z^{k}}{k+1}$, we obtain

$$
t=\alpha^{-1} \sum_{k=0}^{\infty} \frac{\left(\sum_{j=0}^{\infty} v_{j} u^{j}\right)^{k+1}}{(k+1)} .
$$

Based on equations (5.1) and (5.2), we can write

$$
t=\alpha^{-1} \sum_{k, j=0}^{\infty} \frac{c_{k+1, j} u^{j}}{(k+1)}=\sum_{j=0}^{\infty} p_{j} u^{j}
$$

where $p_{j}=\alpha^{-1} \sum_{k=0}^{\infty} c_{k+1, j} /(k+1)$ (for $j \geq 0$ ) and the quantities $c_{k+1, j}$ (for $j=1,2, \ldots$ ) can be determined from the $v_{j}^{\prime}$ s by $c_{k+1, j}=\left(j v_{0}\right)^{-1} \sum_{m=1}^{j}[(k+2) m-j] v_{m} c_{k+1, j-m}$ and $c_{k+1,0}=v_{0}^{k+1}$. Then, from equation (5.3), we obtain

$$
Q(u)=\sum_{i=1}^{\infty} a_{i}\left(\sum_{j=0}^{\infty} p_{j} u^{j}\right)^{i / \gamma} .
$$

Further, we can expand a power quantity $A^{\lambda}$ in Taylor series as $A^{\lambda}=\sum_{k=0}^{\infty}(\lambda)_{k}(A-1)^{k} / k !=$ $\sum_{r=0}^{\infty} f_{r} A^{r}$, where $f_{r}=\sum_{k=r}^{\infty}(-1)^{k-r}\left(\begin{array}{l}k \\ r\end{array}\right)(A)_{k} / k !$ and $(A)_{k}=A(A-1) \ldots(A-k+1)$ is the descending factorial. Then, we can write $Q(u)$ as

$$
Q(u)=\sum_{i=1}^{\infty} \sum_{r=0}^{\infty} a_{i} g_{r, i}\left(\sum_{j=0}^{\infty} p_{j} u^{j}\right)^{r},
$$

where $g_{r, i}=\sum_{k=r}^{\infty}(-1)^{k-r}\left(\begin{array}{l}k \\ r\end{array}\right)(i / \gamma)_{k} / k$ ! Finally, using again (5.1) and (5.2), we obtain (5.6).

\section{References}

[1] Berkson, J. and Gage, R.P. (1952). Survival curves for cancer patients following treatments. Journal of the American Statistical Association, 47, 501-515.

[2] Brys, G., Hubert, M. and Struyf, A. (2006). Robust measures of tail weight. Computational Statistics and Data Analysis, 50, 733-759.

[3] Carrasco, J.M.F., Ortega, E.M.M. and Cordeiro, G.M. (2008). A generalized modified Weibull distribution for lifetime modeling. Computational Statistics and Data Analysis, 53, 450-462.

[4] Cordeiro, G.M., Ortega, E.M.M. and Silva, G.O. (2012). The Kumaraswamy modified Weibull distribution: theory and applications. Journal of Statistical Computation and Simulation, DOI:10.1080/00949655.2012.745125, (print).

[5] Farewell, V.T. (1982). The use mixture models for the analysis of survival data with long-term survivors. Biometrics, 38, 1041-1046.

[6] Gradshteyn, I.S. and Ryzhik, I.M. (2000). Table of integrals, series and products. 6th edition, Edited by Jeffrey, A. and Zwillinger, D. New York, Academic Press.

[7] Ibrahim, J.G., Chen, M.H. and Sinha, D. (2001). Bayesian Survival Analysis. New York, Springer.

[8] Kenney, J.F. and Keeping, E.S. (1962). Mathematics of Statistics. Part 1. New Jersey, Van Nostrand.

[9] Lai, C.D., Xie, M. and Murthy, D.N.P. (2003). A modified Weibull distribution. IEEE Transactions on Reliability, 52, 33-37.

[10] Maller, R. and Zhou, X. (1996). Survival Analysis with Long-Term Survivors. United Kingdom. Chichester, John Wiley.

[11] Mudholkar, G.S., Srivastava, D.K. and Friemer, M. (1995). The exponentiated Weibull family: A reanalysis of the bus-motor-failure data. Technometrics, 37, 436-445. 
[12] Ortega, E.M.M., Rizzato, F.B. and Demtrio, C.G.B. (2009). The generalized log-gamma mixture model with covariates: local influence and residual analysis. Statistical Methods and Applications, 18, 305331.

[13] Seier, E. and Bonett, D. (2003). Two families of kurtosis measures. Metrika, 58, 59-70.

[14] Sy, J.P. and Taylor, M.M.G. (2000). Estimation in Cox a proportional hazards cure model. Biometrics, 56, 227-336.

[15] Xie, M. and Lai, C.D. (1995). Reliability analysis using an additive Weibull model with bathtub-shaped failure rate function. Reliability Engineering and System Safety, 52, 87-93.

[16] Xie, M., Tang, Y. and Goh, T.N. (2002). A modified Weibull extension with bathtub failure rate function. Reliability Engineering and System Safety, 76, 279-285. 